\title{
THE IRISH NATIONAL DEMENTIA STRATEGY
}

Tús Áite do

Shábháilteacht Othar

Patient Safety First
An Roinn Sláinte

DEPARTMENT OF HEALTH 


\section{CONTENTS}

Foreword

Structure of the Strategy

Section 1:- Executive Summary

Section 2:- Background to and Development of the Strategy

Section 3:- Guiding Principles and Priority Actions

Section 4:- Better Awareness and Understanding of Dementia

Section 5:- Timely Diagnosis and Intervention

Section 6:- Integrated Services, Supports and Care for People with Dementia and their Carers

- Integrated Care Pathways

- Primary Care, Mental Health Care and Care in the Community

- Acute Care

- Long Term Care Options

- Palliative Care for People with Dementia

Section 7:- Training and Education

Section 8:- Research and Information Systems

Section 9:- Leadership

Appendices 


\section{Foreword}

We are often reminded that dementia is not a normal part of ageing, but is a disorder that affects many older people, and some younger people also. It is a condition that gives rise to intense fear, perhaps even dread, for many people, and it is often imperfectly understood. Those affected and their families can find it hard to get information, advice and help following a diagnosis of dementia.

Healthcare and community professionals and volunteers can also find it challenging to know how they can best assist someone with dementia, how they should approach diagnosis, and where the person with dementia can be referred on for further assistance and services.

Those with dementia can feel stigmatised and set apart from others because of the poor understanding of dementia among the general public.

This Strategy sets out to improve the lives of people with dementia across all of these fronts. Clear clinical pathways and guidelines, better information about community and other supports, improved linkages between care streams, public awareness, research, and removal of stigma are all addressed. A key message of the Strategy is that with the right supports, a person with dementia can live well, often for quite a long time. The emphasis is therefore on ability rather than on how a person is restricted.

Like any Strategy, particularly one seeking culture change, it will take time to embed and for the desired outcomes to feed through, but clear arrangements are included for implementation and monitoring.

I want to express my sincere thanks to the members of the Working Group who assisted in the preparation of this Strategy, and who so selflessly gave of their time, including repeatedly travelling long distances in some cases, to contribute to this important task. I would also like to extend my gratitude to The Atlantic Philanthropies for their continued assistance and support in this area.

Kathleen Lynch, T.D., Minister for Primary Care, Social Care (Disabilities/Older People) and Mental Health December, 2014 


\section{STRUCTURE OF THE STRATEGY}

\section{Section 1 is the Executive Summary}

Section 2 explains the Background to and Development of the Strategy, which is based on the following six Priority Action Areas:-

- Better awareness and understanding;

- Timely diagnosis and intervention;

- Integrated services, supports and care for people with dementia and their carers;

- Training and education;

- Research and information systems;

- Leadership.

Section 3 sets out Guiding Principles and Priority Actions.

The Guiding Principles are those principles that should inform and underpin services, supports and initiatives across all health and social care streams for people with dementia, their carers, and families.

Priority Actions are considered to be key to implementation of the Strategy and to be capable of implementation within existing resources or by reconfiguring these resources. These Actions are drawn from across all five of the Strategy's Priority Action Areas, and some have relevance across more than one Action Area.

Sections 4 to 9 discuss each of the Priority Action Areas in turn, and include the Objectives of the Strategy in each area and a number of Actions additional to the Priority Actions in Section 3. The Actions in this Section may, in some cases, require resources additional to what is currently available, and will be considered as the required resources become available in the future. 


\section{SECTION 1 \\ EXECUTIVE SUMMARY}

A commitment to develop a Dementia Strategy is included in the Programme for Government 2011-2016. The Strategy was prepared with the assistance of an expert Working Group which included Clinicians and other healthcare professionals, researchers, and representatives of the Department of Health and the Health Service Executive.

Membership of the expert Working Group was as follows:-

- Mr Barry Murphy, Principal Officer, Services for Older People, Department of Health;

- Mr Noel Mulvihill, Assistant National Director for Older Persons, Health Service Executive;

- Dr Caitriona Crowe, Consultant in Old Age Psychiatry, South Tipperary Mental Health Service Executive, Clonmel;

- Dr Tony Foley, General Practitioner, Kinsale, Member of KCoRD \& Lecturer, Department of General Practice, UCC;

- Mr Gerry Martin, CEO, The Alzheimer Society of Ireland;

- Mr Maurice O'Connell, former CEO, The Alzheimer Society of Ireland;

- Ms Eilis Hession, Project Lead, Genio Living Well with Dementia Project, and Manager, Services for Older People, Health Service Executive, Dún Laoghaire;

- Associate Prof. Suzanne Cahill, Director, Dementia Services Information and Development Centre, St James' Hospital and School of Social Work and Social Policy, TCD;

- Dr Kate Irving, Lecturer in Mental Health Nursing, DCU;

- Dr Fiona Keogh, Director of Research and Evidence, Genio;

- Dr Shaun O'Keeffe, Consultant in Geriatric and General Medicine, Merlin Park University Hospital, Galway.

Secretariat

- Ms Sinéad Quill, Assistant Principal, Services for Older People, Department of Health;

- Ms Joanne Clarke, Staff Officer, Services for Older People, Department of Health.

The Strategy identifies key principles to underpin and inform the full range of health and social care services provided to people with dementia, their families and carers. These include the following:-

- An integrated, population-based approach should be taken to dementia service provision;

- Services, including palliative care services, should be tailored to deliver the best possible outcomes for people with dementia and their families and carers;

- All communications with those with dementia should be as accessible as possible;

- All those dealing with people with dementia across health and social care settings should be appropriately trained. 
Priority Actions which are considered to be capable of delivery within existing resources, or by reconfiguring the resources which are currently available, have been identified, and are broadly based on the following:-

- Clear responsibility for dementia will be assigned within the Health Service Executive, both at management level and at the front-line, to manage the progress of individual patients through their care journey;

- Clear descriptions of care pathways will be prepared, and clear information and effective assistance will be made available to GPs and to people with dementia and their families and carers to identify and access available services and supports;

- Measures will be taken to promote a better understanding of dementia, including modifiable risk factors, and to remove the stigma sometimes associated with the condition;

- The way in which resources are deployed and services configured will be reviewed to ensure that available resources are used to optimal effect;

- Research to inform the design and delivery of dementia services in Ireland will be supported and given appropriate priority.

Priority Action Areas are discussed in some detail and a number of additional Actions, some of which may require additional resources as and when these become available, are also identified. 


\section{SECTION 2 \\ BACKGROUND TO AND DEVELOPMENT OF THE STRATEGY}

The Programme for Government 2011-2016 commits to developing:

'a national Alzheimer's and other dementias strategy to 1) increase awareness 2) ensure early diagnosis and intervention and 3) develop enhanced community based services'.

The aim of the Strategy is to improve dementia care so that people with dementia can live well for as long as possible, can ultimately die with comfort and dignity, and can have services and supports delivered in the best way possible.

\section{How was the Strategy developed?}

The following informed the development of this Strategy:

1. Research review

2. Review of international dementia policies/plans/strategies

3. Review of Irish policy contexts

4. Public consultation process

5. Clinicians' Roundtables (2) on the National Dementia Strategy

6. Workshops (2) with people with dementia and carers

7. National Dementia Strategy Advisory Group

\section{Research Review}

Creating Excellence in Dementia Care: A Research Review for Ireland's National Dementia Strategy (Cahill et al. 2012) was funded by The Atlantic Philanthropies to:

- review current and future demographic trends in Ireland and provide estimates of current and future dementia prevalence, for those aged 65 and over and for younger people;

- specify the main economic costs of dementia care;

- review current service availability (based on the recent Health Service Executive (HSE) audit) and estimated future demand for services;

- review best practice in dementia care locally and internationally.

The Strategy draws in particular on this research.

\section{Review of international dementia policies/plans/strategies}

While the emphasis varies across international dementia policies, plans and strategies, it was possible to identify a number of common elements, including:-

- Prevention;

- Timely diagnosis; 
- Training and education for care providers;

- Information, raising awareness, enhancing understanding among general public;

- Supports for carers;

- Co-ordination and collaboration between all stakeholders (service providers, carers, people with dementia);

- Quality service provision in all settings (community/acute/residential care/end-of-life care);

- Research;

- Governance/leadership.

\section{Review of Irish policy contexts}

Until now no specific policy for dementia has been developed in Ireland, but a number of reports and policy initiatives had direct relevance for the development of the Dementia Strategy. These are referenced throughout the Strategy.

\section{Public consultation process}

A public consultation process to inform the development of the Strategy took place during July/August 2012, with 73 responses received.

A report of the consultation process is available at

http://www.dohc.ie/publications/NatStratDementia_SummaryConsultation.html

\section{Clinicians' Roundtables on the National Dementia Strategy}

Convened by the Alzheimer Society of Ireland, representatives of Old Age Psychiatry, Geriatric Medicine, Neurology and Psychology and other healthcare professionals had two Roundtable discussions on relevant issues.

Reports of these Roundtables are available at http://www.alzheimer.ie/Alzheimer/media/SiteMedia/ImageSlider/Fixed/ASI_CliniciansRoundtable_Report-2013.pdf;

http://www.alzheimer.ie/Alzheimer/media/SiteMedia/Userlmages/Orchard/ Multidisciplinary-Clinicians-Roundtable_Report_June-2013.pdf

\section{Workshops (2) for People with Dementia, Carers and Former Carers}

Workshops were convened by the Alzheimer Society of Ireland to allow people with dementia and their carers, along with former carers, to meet with the Strategy Working Group.

A summary report of these Workshops is available at http://www.alzheimer.ie/Alzheimer/media/SiteMedia/ImageSlider/Fixed/Living-withDementia_NDS-Roundtable-report_September-2013_The-Alzheimer-Society-of-Ireland.pdf 


\section{What is Dementia?}

Dementia is a syndrome characterised by progressive cognitive impairment and is associated with impairment in functional abilities and, in many cases, behavioural and psychological symptoms. There may be memory loss, usually related to short-term memory, communication difficulties, changes in personality or mood and problems with spatial awareness.

Many different diseases can produce the symptoms of dementia. However, Alzheimer's disease accounts for a majority of cases, while Vascular Dementia is the second most common dementia sub-type.

Other less common causes include mixed dementia (Alzheimer's Disease and Vascular Dementia), Dementia with Lewy Bodies, Frontotemporal dementia (FTD), Korsakoff's Disease, Huntington's Disease, Creutzfeldt-Jakob Disease (CJD) and HIV-associated dementia (HAD). The different types of dementia are associated with particular types of brain cell damage in particular regions of the brain.

Despite the strong association between dementia and old age, it is not solely a disease of old age. There are significant numbers within the overall dementia population with early onset dementia. People with Down Syndrome face an increased risk in this regard. Currently, it is estimated that there are approximately 4,000 people under the age of 65 years with dementia in Ireland.

While dementia can bring many challenges both for the person with dementia and people caring for them, there is now an increasing recognition that people can live well with dementia for a number of years, as long as there is timely access to an assessment and diagnosis and the right services and supports are in place.

The prospective ageing of the Irish population will lead to an exponential increase in the number of people with dementia in the years ahead ${ }^{1}$. Based on CSO population projections over the next 30 years and applying the EuroCode age/gender-specific dementia prevalence rates, the following Table shows the expected increase in the numbers of people likely to present with dementia in Ireland.

\footnotetext{
${ }^{1}$ Despite a number of lifestyle and genetic risk factors contributing to the different types of dementia, increasing age remains the strongest risk factor, with prevalence rates nearly doubling every five years over the age of 65 years.
} 
Estimated number and projected growth in the number of people with dementia in Ireland by age group, 2011-2046

\begin{tabular}{|c|r|r|r|r|r|r|r|r|}
\hline Age group & $\mathbf{2 0 1 1}$ & $\mathbf{2 0 1 6}$ & $\mathbf{2 0 2 1}$ & $\mathbf{2 0 2 6}$ & $\mathbf{2 0 3 1}$ & $\mathbf{2 0 3 6}$ & $\mathbf{2 0 4 1}$ & $\mathbf{2 0 4 6}$ \\
\hline $\mathbf{3 0 - 5 9}$ & 2,866 & 2,935 & 2,934 & 2,869 & 2,854 & 2,864 & 2,889 & 2,991 \\
\hline $\mathbf{6 0 - 6 4}$ & 1,200 & 1,301 & 1,449 & 1,615 & 1,738 & 1,906 & 2,044 & 1,896 \\
\hline $\mathbf{6 5 - 6 9}$ & 2,776 & 3,287 & 3,827 & 4,020 & 4,485 & 4,876 & 5,315 & 5,645 \\
\hline $\mathbf{7 0 - 7 4}$ & 4,604 & 5,532 & 7,013 & 7,442 & 8,367 & 9,378 & 10,211 & 11,188 \\
\hline $\mathbf{7 5 - 7 9}$ & 7,475 & 8,213 & 11,298 & 12,560 & 14,055 & 15,928 & 17,968 & 19,692 \\
\hline $\mathbf{8 0 - 8 4}$ & 10,958 & 12,265 & 16,099 & 17,868 & 22,348 & 25,364 & 29,102 & 33,196 \\
\hline $\mathbf{8 5 +}$ & 17,970 & 21,260 & 25,595 & 31,085 & 40,195 & 52,512 & 64,654 & 77,549 \\
\hline Total & 47,849 & 54,793 & 68,216 & 77,460 & 94,042 & 112,828 & 132,182 & 152,157 \\
\hline
\end{tabular}

Note: Estimates for 2011 based on Census of Population 2011. Projections for 2016 to 2041 based on CSO (2013) Population and Labour Force Projections, 2016-2014, Stationery Office, Dublin, Table 3, page 40 and EuroCoDe (2009) Age and gender specific dementia prevalence rates. (Source: Pierce, M. et al. (forthcoming). Prevalence and Projections of Dementia in Ireland, 2011. Genio Ltd., Mullingar).

It has been estimated that the average annual cost per person with dementia in Ireland is $€ 40,500,{ }^{2}$ with the bulk of care for people with dementia being provided free of charge by family caregivers, many of whom are adult children and spouses. The value of informal care for those with dementia is estimated to be $€ 807$ million per annum.

While dementia is associated with increasing age and usually begins to present in the population after the age of 65 , people in their 30 s, 40 s or 50 s can experience it. The diagnosis of younger onset dementia is challenging, with symptoms often confused with other disorders and disabilities, such as depression and other mental health problems. People with younger onset dementia are most commonly affected by Alzheimer's Disease, Vascular Dementia and Dementia with Lewy Bodies.

Some develop younger onset dementia alongside other disorders such as Down Syndrome, Parkinson's disease, Acquired Immune Deficiency Syndrome (AIDS), Huntington's disease, Creutzfeldt-Jakob disease, and alcoholism.

The difficulties experienced by younger people with dementia are compounded by the fact that many are still employed in the labour market and will have financial responsibilities including mortgages. Many will also have parental and family responsibilities. People with early onset dementia usually experience greater difficulty accessing a diagnosis and fitting into existing dementia service provision, which is generally tailored to the needs of older people.

This Strategy addresses the needs of all people with dementia, including those with younger onset dementia.

\footnotetext{
${ }^{2}$ https://www.tcd.ie/Communications/content/pdf/Creating_Excellence_in_Dementia_Care2012.pdf
} 


\section{SECTION 3 GUIDING PRINCIPLES AND PRIORITY ACTIONS}

\section{Principles underpinning the Strategy}

The Irish National Dementia Strategy seeks to progress the dual and overarching principles of personhood and citizenship by enabling people with dementia to maintain their identity, resilience and dignity and by recognising that they remain valued, independent citizens who, along with their carers, have the right to be fully included as active citizens in society.

Future Health (A Strategic Framework for Reform of the Health Service 2012- 2015) commits to caring for more people in their homes for as long as possible, rather than in residential care. ${ }^{3}$ It commits to a new concentration on keeping people healthy throughout their lifecourse, and to treating patients at the lowest level of complexity that is safe, timely, efficient and as close to home as possible. It commits to a patient-centred, flexible, community-based service. It commits to including natural supports (such as family, friends and social interactions) as much as possible. In this Strategy these commitments are reaffirmed, and all of those involved in designing and delivering services and supports to people with dementia must take account of these principles on an ongoing basis.

Dementia policy, service delivery and development should be guided by the principles of chronic disease management set out in the Department of Health's Policy Framework for the Management of Chronic Diseases. ${ }^{4}$ These include:-

- A national focus on population-directed health promotion;

- The development of structured, planned care for patients with long-term chronic conditions;

- The use of information systems and registers to plan and evaluate care for individuals with chronic disease;

- The development of a model of shared care that is integrated across organisational boundaries;

- Planning care that is delivered in the appropriate setting;

- Using multidisciplinary teams in the provision of care;

- Providing a monitoring and evaluation framework for chronic disease programmes.

\footnotetext{
${ }^{3}$ http://health.gov.ie/blog/publications/future-health-a-strategic-framework-for-reform-of-the-healthservice-2012-2015/

${ }^{4} \mathrm{http}: / /$ www.hse.ie/eng/About/Who/Population_Health/Population_Health_Approach/Population_Health_ Chronic_illness_Framework_July_2008.pdf
} 
This Dementia Strategy identifies a number of additional principles which should similarly underpin all approaches to care and support for those with dementia.

The Strategy seeks to translate these principles into practice through the refocusing of current service delivery to address the needs of people with dementia and their carers in a way that is responsive and flexible. Recognising that a social and clinical response is required and that dementia cuts across many areas of provision, the Strategy also emphasises the need for a 'whole community response' to dementia, with health and social care services as only one part of the package of supports that people with dementia need.

\section{Key Principles}

1. Appropriate account should be taken of dementia in the development and implementation of existing and future health policies.

2. The approach of the Age Friendly Cities and Counties Programme and the Healthy Communities/Cities Programme, which is to enable people with dementia to live well as valued citizens, should be supported and encouraged, including through representation for people with dementia on Older People's Councils, the establishment of which has been committed to in the Programme for Government.

3. Regular assessments of palliative care need should be conducted by staff providing care to people with dementia. Staff should have the training in the principles of palliative care needed to assess palliative care need, and referral should be made to specialist palliative care services to support care provision where required. People with dementia should be supported to be cared for in the place of their choice, as far as is possible, including at the end of life.

4. Those who provide services/care or who interact with people with dementia, including healthcare professionals, clerical and administrative staff, volunteers and staff of external organisations, should be appropriately trained and regularly updated in dealing and communicating with those with dementia. All communications with, or material relevant to, people with dementia should have content and format that maximises accessibility and ease of understanding.

5. People with dementia should be supported to retain skills as much as possible.

6. Available resources should be deployed on the basis of need and as effectively as possible to provide services for all people with dementia, including those with earlyonset dementia and/or an intellectual disability, and should be delivered in a culturally appropriate way.

7. An integrated partnership approach should be taken to the planning, development, delivery, evaluation and monitoring of services for people with dementia, with the inclusion of all stakeholders from the public (i.e. local authorities and other state agencies, health and social services), private and voluntary sectors. 
8. Clinical effectiveness as a key component of safe, quality care is an underpinning principle of the National Dementia Strategy. A clinical effectiveness approach incorporating national and international best evidence will promote the delivery of integrated dementia care that is current, effective and consistent.

\section{Priority Actions}

\section{Better Awareness and Understanding}

1. Public awareness and health promotion measures will be implemented by the Health Service Executive in order to:-

- provide a better understanding of dementia in society generally;

- reduce the stigma that can be associated with dementia;

- target populations particularly at risk, including people with an intellectual disability;

- support the implementation of Healthy Ireland $(2013)^{5}$ by highlighting the modifiable lifestyle and cardiovascular risk factors which can beneficially impact on risk and time of onset of dementia, and by implementing the National Physical Activity Plan (in preparation) which will encourage the population to be more physically active.

\section{Timely Diagnosis and Intervention}

2. The Health Service Executive will develop a National and Local Dementia Care Pathway to describe and clearly signpost the optimal journey through the system from initial presentation with worrying symptoms, through to diagnosis, including levels of intervention appropriate to need at any given time.

3. The following material will be developed and made available to GPs:-

- Dementia-specific reference material to broaden skills base;

- Guidance on national and local pathways to investigation and diagnosis;

- Information about health and social supports available in the local community.

4. The Health Service Executive will develop guidance material on the appropriate management of medication for people with dementia, and in particular on psychotropic medication management, and make arrangements for this material to be made available in all relevant settings, including nursing homes.

5. Modifiable lifestyle risk factors, such as tobacco and alcohol use and physical inactivity, should be actively managed as part of the care plan for people with dementia.

6. The Health Service Executive will review existing service arrangements so as to maximise the access that GPs and acute hospital clinicians have to specialist assessment and diagnosis of dementia, including Old Age Psychiatry, intellectual disability services, geriatric medicine, neurology services and memory clinics.

\footnotetext{
${ }^{5}$ http://www.hse.ie/eng/services/publications/corporate/hieng.pdf
} 


\section{Integrated Services, Supports and Care for People with Dementia and their Carers}

7. The Health Service Executive will critically review health and personal social services for people with dementia to:-

- identify gaps in existing provision, and

- prioritise areas for action in accordance with resource availability, with priority being given to the most urgent deficits that can be addressed either within or by reconfiguring existing resources.

8. The Health Service Executive will consider how best to configure resources currently invested in home care packages and respite care so as to facilitate people with dementia to continue living in their own homes and communities for as long as possible and to improve the supports available for carers.

9. The Health Service Executive will evaluate the potential of assistive technology to provide flexible support both to carers and to people with dementia.

10. In line with the health promoting health service model, the Health Service Executive will ensure that information on how to access advocacy services, voluntary organisations and other support services is routinely given to people with dementia and their families/ carers.

\section{Training and Education}

11. The Health Service Executive will engage with relevant professional and academic organisations to encourage and facilitate the provision of dementia-specific training, including continuous professional development, to relevant occupational and professional groups, including peer-led support and education for GPs, and to staff of nursing homes.

\section{Leadership}

12. Clear overall responsibility for dementia will be assigned to a person at senior management level within the Health Service Executive.

13. The Clinical Strategy and Programmes Division of the Health Service Executive will establish a Workstream on Dementia Care as part of its Integrated Care Programme for Older Persons, in recognition of the complexity of the illness and the need for leadership and integration across all relevant Health Service Executive Directorates.

14. Within primary care services, a named key worker will be be appointed to play a key role in co-ordinating each patient's care and promoting continuity, and ensuring that the patient knows who to access for information and advice. 


\title{
SECTION 4 \\ BETTER AWARENESS AND UNDERSTANDING OF DEMENTIA
}

\begin{abstract}
Stigma and misunderstanding can have a devastating impact on all stages of a person's journey through dementia (WHO, 2012)
\end{abstract}

\section{OBJECTIVES}

More humane, well-informed, positive public attitudes to dementia, leading to reduced stigma and to increased readiness in communities to support people with dementia.

Increased awareness and better understanding of brain health, dementia and the overall consequences (positive and otherwise) of receiving a timely diagnosis of dementia.

While many of us know someone with dementia, the general public's understanding of the illness is low. Dementia is often incorrectly understood as being a normal part of growing older rather than an organic brain disease. Many are not aware of the range of symptoms other than memory problems associated with dementia. What many people understand to mean "dementia" corresponds to the later stages of the disease. The helplessness associated by many with advanced dementia brings with it a fear and a stigma which makes the lived experience of people with dementia even worse.

While dementia care can be extremely challenging, particularly for family caregivers, the knowledge that people with dementia can live well in the community, with the necessary statutory, voluntary and community supports in place, may encourage those experiencing early signs and symptoms to come forward for diagnosis and support. It may also reduce any reluctance among health practitioners to make and communicate a diagnosis. Better awareness facilitates enhanced involvement with and support from the wider community. It gives carers, family members and friends an understanding and appreciation of the situation of the person affected by dementia and it reduces fear for all involved.

The need to challenge the stigma associated with dementia was highlighted on a number of occasions during the consultation process.

In a major report on the stigma that can be associated with dementia, Alzheimer's Disease International $(2012)^{6}$ made ten key recommendations:-

- Educate the public;

- Reduce isolation of people with dementia;

- Give people with dementia a voice;

- Recognise the rights of people with dementia and their carers;

${ }^{6} \mathrm{http}: / /$ www.alz.co.uk/research/WorldAlzheimerReport2012.pdf 
- Involve people with dementia in their local communities;

- Support and educate informal and paid carers;

- Improve the quality of care at home and in care homes;

- Improve dementia training of primary healthcare physicians;

- Call on governments to create national Alzheimer's disease plans;

- Increase research into how to address stigma.

Organisations such as the Alzheimer Society of Ireland (ASI) and the Dementia Services Information and Development Centre (DSIDC) have, for a number of years, played a critical role in promoting awareness and challenging stigma. The ASI has focused on creating awareness among the general public. The DSIDC focuses on healthcare professionals' awareness and training about dementia.

\section{Awareness of modifiable risk factors for dementia}

Although no cure for dementia is currently available, research has found that there are a number of ways to decrease the risk of getting dementia or delay the age of onset. It has been reported that, by delaying onset by two years, prevalence rates would be reduced by $20 \%$ and by delaying onset by five years, prevalence rates could be halved.

Evidence now suggests that lifestyle factors which impact on cardiovascular health (e.g. smoking, obesity, and physical inactivity) can also impact on dementia. Diabetes and hypertension impact on dementia risk, so modifiable behaviours which contribute to these conditions are also relevant to dementia. Social isolation and levels of cognitive activity seem to play a part too.

Non-modifiable risk factors associated with dementia include age, gender and genetics.

The need for primary prevention and better public awareness featured strongly in the public consultation process.

\section{DEMENTIA-FRIENDLY COMMUNITIES}

More supportive attitudes in society, regarding people with dementia first and foremost as people, for whose particular needs adaptations may need to be made just as they are for many other groups, would make it much easier for people with dementia to continue to participate in society. In turn, increased involvement in society may help to normalise dementia in a way that is long overdue (Nuffield Council on Bioethics, 2009).?

\footnotetext{
${ }^{7}$ http://nuffieldbioethics.org/wp-content/uploads/Dementia-Chapter-4-Dementia-and-society.pdf
} 
Because dementia requires both a medical and a societal response, a "whole community" approach is needed, particularly in the earlier stages of the condition. Communities are key to ensuring that staying at home with dementia is a feasible option. A combination of formal and informal supports offers the best opportunity to produce the optimal outcome for the person with dementia and their carers. Examples of community supports currently available for people with dementia include Alzheimer Cafés, social clubs, arts-based activities, family carer training and peer-led support groups.

The Irish Age Friendly Cities \& Counties Programme is a national initiative aimed at improving quality of life for older people at local level. Under the Programme, key public, private and voluntary sector agencies come together under the aegis of the Local Authorities to ensure that their combined resources are used optimally in delivering necessary services to older people. The Age Friendly Programme includes the establishment of Older People's Councils where older people can raise issues of concern. It is expected that all Local Authorities will have adopted, or committed to adopting, the Age Friendly Programme by the end of January 2015.

The Alzheimer Society of Ireland (ASI) is leading a Dementia Friendly Communities (DFC) initiative that aims to transform villages, towns, cities and communities into better places to live for people with dementia. There are now seven communities of interest across the country being supported to deliver a range of DFC activity including community education, developing volunteering, supporting local business to become dementia friendly and improving the social and physical environments.

Dementia Friendly Communities seek to encourage more collaboration between nongovernmental organisations (NGOs) and local services, and ensure that there is a better awareness, understanding, and sensitivity throughout the local community about the needs of people with dementia.

During the consultation process, respondents noted the key role that communities should play in normalising the lived experience of people with dementia.

PRIORITY ACTION 1 IN SECTION 3 RELATES TO THIS ACTION AREA

\section{ADDITIONAL ACTION}

The Department of Health and the Health Service Executive will consider how best to promote a better understanding of and sensitivity to dementia among staff of frontline public services, as part of the Health-Promoting Health Service Executive Initiative. 


\section{SECTION 5 \\ TIMELY DIAGNOSIS AND INTERVENTION}

Contrary to social misperception, there is a very great deal that can be done to help people with dementia. Services need to be re-engineered so that dementia is diagnosed early and well and so that people with dementia and their family carers can receive the treatment, care and support following diagnosis that will enable them to live as well as possible with dementia (Department of Health (UK), 2009) ${ }^{8}$

\section{OBJECTIVES}

People receive a timely diagnosis of dementia and dementia sub-type.

People know to approach their GP if they have concerns about their cognitive health.

GPs are supported to assess, diagnose and to provide information on brain health and on dementia as necessary.

Healthcare professionals advise people who have concerns about their cognitive health to go to their GP.

Clear information on accessing assessment and diagnostic services to be available to GPs, in the first instance, and then to other health and social care professionals.

As much specialist expertise as possible to be available regionally to ensure appropriate investigation and timely diagnosis of dementia in primary, mental health, acute and long term care services.

Sensitive disclosure of a diagnosis and discussion of dementia, taking an individualised and person-centred approach.

Following a diagnosis, people with dementia and their carers know where to seek help or support, who to talk to about accessing services or entitlements and where to go for information.

Availability of a range of flexible, community-based supports that draw on and integrate community and voluntary organisations and mainstream services as well as health and social care services.

\footnotetext{
${ }^{8}$ https://www.gov.uk/government/uploads/system/uploads/attachment_data/file/168220/dh_094051.pdf
} 


\section{Timely diagnosis}

A timely diagnosis is one that is made and communicated at a time and in a way that best matches the physical, emotional, medical, and other needs of the patient, their families, and carers.

Dementia is, by and large, irreversible but a diagnosis can facilitate a better understanding of the illness and open a channel to information, supports and medication.

A recent systematic review has found that there is no evidence that general population screening for dementia would lead to improved clinical or psychosocial outcomes. Clinical guidelines are unequivocal in this regard. There may however be merit in targeted screening for persons with an intellectual disability, including Down Syndrome given the high rate/typically early age of onset of dementia in this population. The value of establishing a reliable baseline for these groups will be examined further by the Department of Health.

The General Practitioner (GP) is usually the first contact when concerns about thinking or memory arise. The GP role involves identifying those with symptoms that may indicate dementia, excluding any other possible diagnoses and referring on to specialist services. GPs will also have an ongoing role supporting patients and their families throughout their illness.

However, some of the difficulties identified by GPs in diagnosing dementia include:-

- difficulty differentiating normal ageing from signs and symptoms of dementia;

- lack of confidence;

- the belief that nothing can be done for someone with dementia;

- concerns about the impact of the diagnosis on the patient;

- fear of making a wrong diagnosis.

A dementia reference guide for GPs entitled "Dementia - Diagnosis \& Management in General Practice"was published by the Irish College of General Practitioners in 2014. ${ }^{9}$ This document focuses on key aspects of dementia care including timely diagnosis, early intervention, activation of social supports, multidisciplinary approaches, legal issues and the appropriate use of medication in primary care.

While GPs play a key role in diagnosis, a confirmatory definitive diagnosis and identification of the dementia sub-type remains a specialist task. Accurate diagnosis and subtyping has become more important for a number of reasons, including the ability to tailor treatments for Alzheimer's Disease and Vascular Dementia and because of the need to avoid the potentially serious side-effects of antipsychotic drug use in people with Dementia with Lewy Bodies. The earlier the stage of dementia, the more difficult it is to make this diagnosis. The same is true for atypical or complex presentations and cases presenting under 65 years. Neurologists, Geriatricians, Old Age Psychiatrists and Memory Clinics all have a role in dementia diagnosis and subtyping.

\footnotetext{
${ }^{9}$ http://www.icgp.ie/go/library/catalogue/item/7BA666D7-BE51-45F2-138D256A9F394D34
} 
Where Memory Clinics are available, referral pathways will be clarified, with agreed GP workup, use of common referral templates and appropriate triage of referrals. These measures will ensure that patients receive an appointment and access to specialist opinion in as timely a manner as possible. Old Age Psychiatry plays a key role at all stages of dementia care, from detection, assessment and diagnosis, right through to care for people with dementia of all types, at all stages of the illness trajectory and in all clinical contexts.

During the consultation process, there was a general consensus that timely diagnosis is essential for ensuring timely interventions. Respondents highlighted the need to develop a structured and standardised education programme for health professionals in this regard. A number of respondents stressed the need for systems, structures and age appropriate services specifically to promote timely diagnosis of people experiencing early onset dementia, including people with Down Syndrome.

Following a diagnosis, the person with dementia will need considerable emotional and practical support. Local, well-co-ordinated support services are needed to assist people and their families to cope with the choices and dilemmas confronting them at this often distressing and challenging time.

Availability of relevant information and guidance can greatly assist those who have dementia and those who care for them. The information needs of people with dementia and their families include:-

- the signs and symptoms of dementia;

- its course and prognosis;

- medications;

- local care and support services;

- support groups;

- sources of financial and legal advice, and advocacy;

- the income supports available for those with an illness/disability and for those with caring responsibilities ${ }^{10}$;

- medico-legal issues, including driving; and

- local information sources, including libraries and voluntary organisations.

There is uncertainty among people with dementia and their carers about where to seek help or support, who to talk to about living and coping on a daily basis with dementia, about where to access services or entitlements and about where to go for information.

The Alzheimer Society of Ireland rolled out a Dementia Advisor programme in July 2014, employing seven Dementia Advisers across the country. ${ }^{11}$ Their specific mandate is to provide

\footnotetext{
${ }^{10}$ The Department of Social Protection provides a range of payments and supports that people with dementia might qualify for, including State pensions and illness and disability payments, such as Illness Benefit, Disability Allowance or the Invalidity Pension. Workplace supports and payments to facilitate participation by people with disabilities in the labour force include the Partial Capacity Benefit and the Wage Subsidy scheme. In addition, family carers may qualify for Carer's Allowance or Carer's Benefit and the Respite Care grant. Further information is available at www.welfare.ie

${ }^{11}$ http://www.alzheimer.ie/Services-Support/About-our-Services/Dementia-Advisor.aspx
} 
support and information to people with dementia and their carers living in the community. They will have a strong presence on the ground and work closely with Primary Care Teams, keyhealth and social care professionals and service providers locally in order to signpost people to the resources that are available locally as well as provide timely information and social support.

It is not appropriate simply to assume that people with dementia are unable to make their own decisions. The independence of individuals should be protected for as long as possible and, as far as possible, according to their will and preferences. It is intended that the Assisted Decision-Making (Capacity) Bill 2013, when enacted, will support and protect people with dementia who lack or will shortly lack capacity to make and communicate decisions. ${ }^{12}$

\section{Supporting carers of people with dementia}

Ireland's first National Carers' Strategy was published in $2012^{13}$ :-

- to progress the development of supports and services;

- to recognise and respect carers as key partners and to support them to maintain their own health and wellbeing;

- to enable carers to care with confidence;

- to empower them;

- to enable them to participate as fully as possible in economic and social life.

The majority of people with dementia live in their own communities and carers are the cornerstone of care for these people. Dementia is an illness which places huge demands on family caregivers and can adversely affect carers' health, psychological well-being, finances, social life and the carer/care-recipient relationship. Apart from being very stressful for the primary caregiver, caring can seriously affect other family members, including children, grandchildren and siblings. Furthermore, given the degenerative nature of dementia, care is characterised by continuous adjustment and adaptation. Unlike many other forms of informal care, caring for a relative with dementia can extend around the clock. A survey of Alzheimer Society of Ireland family caregivers indicates that, of the individuals caring for a person in middle to late stages of dementia, $69 \%$ will be providing 14 or more hours per day of care. ${ }^{14}$

Services such as day care, home help and particularly home care packages can clearly assist family caregivers to continue providing care, but their availability is uneven. Given the intensive nature of caring for someone with dementia, particularly during its later stages, respite services which are responsive and tailored to the needs of the person with dementia as well as the carer can make a vital contribution to achieving the best possible outcomes for all.

\footnotetext{
${ }^{12} \mathrm{http}: / /$ www.oireachtas.ie/documents/bills28/bills/2013/8313/b8313d.pdf

${ }^{13}$ http://health.gov.ie/blog/publications/national-carers-strategy/

${ }^{14}$ https://www.alzheimer.ie/Alzheimer/media/SiteMedia/An-Economic-Perspective-of-Dementia-Care-inIreland-\%28full-version\%29.pdf?ext=.pdf
} 
The Health Service Executive is committed to implementing a Single Assessment Tool (SAT) for older people to assess need and allocate services and supports in the most effective way possible. The SAT will include provision for assessment of the position and needs of carers as well as of those being cared for.

Assistive technology ranges from very simple tools, such as calendar clocks and touch lamps, to high-tech solutions such as satellite navigation systems to help find someone who has gone missing. These modifications and technologies can promote independence and autonomy, both for the person with dementia and those around them, and can reduce the stress on carers, improving their quality of life, and that of the person with dementia. The consent of the person with dementia should, where possible, be sought and obtained for the use of assistive technology.

It must be noted that there are limitations to what assistive technologies can achieve, and there are a range of ethical issues that must be considered. The person with dementia should be involved in the decision to use assistive technology and their consent always sought and given, where possible.

\section{PRIORITY ACTIONS 2 TO 6 IN SECTION 3 RELATE TO THIS ACTION AREA}

\section{ADDITIONAL ACTIONS}

The Health Service Executive will implement the National Consent Policy (Health Service Executive - May 2013). ${ }^{15}$

The Health Service Executive will promote an awareness of the Assisted Decision-Making (Capacity) Bill, when enacted, to ensure that people with dementia are supported to participate in all decisions that affect them, according to their will and preference.

The Health Service Executive will consider the provision of Dementia Advisors on the basis of the experience of demonstrator sites, with an appropriate number of such Advisors to be dedicated to the needs of those with early-onset dementia.

The Health Service Executive will examine the issues arising regarding the assessment of those with Down Syndrome and other types of intellectual disability given the early age of onset of dementia for these groups and the value of establishing a reliable baseline.

\footnotetext{
${ }^{15}$ http://www.hse.ie/eng/about/Who/qualityandpatientsafety/National_Consent_Policy/National\%20 Consent\%20PolicyMay14.pdf
} 


\section{SECTION 6 \\ INTEGRATED SERVICES, SUPPORTS AND CARE FOR PEOPLE WITH DEMENTIA AND THEIR CARERS}

\section{OBJECTIVES}

Better availability of a range of person-centred, flexible and responsive services that encourage people with dementia to make decisions around their care and that respect their will and preferences from diagnosis through to end-of-life care.

Better co-ordination, integration and smoother transitions between primary, secondary, mental health, community care, acute, long term and palliative care services.

National and local care pathways that signpost people with dementia and their carers and health and social care professionals to the right care, in the right place and at the right time.

People with dementia should be enabled and assisted to access and navigate the health and social care system.

People with dementia should be facilitated to remain living in their own homes and to maintain existing roles and relationships for as long as possible, supported by the wider community along with primary, secondary and community care services. As much support as possible should be given to informal and family carers who care for people with dementia at home.

An integrated and multi-disciplinary response by community services, primary care and secondary care to support people with dementia and their carers.

Hospitals should be dementia-friendly from admission to discharge/death. This includes environmental aspects as well as clinical support.

When admission to acute care is necessary, people with dementia should be treated and supported in an expeditious manner, spending no longer than is absolutely necessary in an acute bed and discharged back into the community.

Staff in all care settings should have the necessary training for treating and supporting a person with dementia, including training in palliative approaches that are appropriate for people with dementia.

There should be a range of long-term care options designed to comply with best practice architectural principles and staffed by competent and skilled personnel trained to address the complex and unique needs and preferences of people with dementia of all ages. 
People who develop dementia in long-term care should be provided with the same opportunities for diagnosis, treatment and care as those in other care settings.

People with dementia should be facilitated and supported to live and die well in their chosen environment including their own home or nursing home if that is their choice. Specialist palliative care should be available to all people with dementia and their families in all care settings and at home, if required, to optimise quality of life and support a comfortable and dignified death

\section{Integrated care pathways}

A person with dementia and their carer(s) need a clearly signposted pathway that directs them to the right care and support, in the right place and at the right time.

During the consultation process, the need for a clear and integrated care pathway across all services was considered as vital to delivering effective, efficient and person-centred care.

It is also desirable that people with dementia and their carers have a designated contact person (or Case Manager) to help to co-ordinate individual care plans and assist people to navigate the complex care pathways and range of services.

Case management differs from the role of a Dementia Advisor in that case management manages individual cases/caseload through the health and social care system, whereas the Dementia Advisor is a signposting and advisory service.

\section{Primary Care, Mental Health Care and Care in the Community}

The majority of people with dementia live in their own homes. Particularly in the case of people with behaviours that challenge and younger-onset dementia, caring for a person with dementia at home will over the course of their condition require an increasing range of individually tailored and integrated responses from the Old Age Psychiatry service and the Primary Care Team, including allied healthcare professionals.

Public health nurses, community mental health nurses and intellectual disability nurses, home helps, occupational therapists, social workers, physiotherapists, speech and language therapists, pharmacists, day care services, a range of respite services (including in home and residential, elective and emergency), meals-on-wheels and home care packages all have an important part to play in supporting people with dementia to remain viably and safely in their own homes for longer.

The availability of service providers trained in providing care to people with dementia, (including those with younger-onset dementia, sensory disability, intellectual disability, behaviours that challenge, and early onset dementia) is very important. It is critical that equity apply to service provision, and that the importance of enablement and reablement underpin decisions on the nature of service provided. 


\section{Acute Care}

Estimates suggest that up to $29 \%$ of all patients in a typical general hospital may have dementia. The cost of dementia care in acute hospitals in Ireland has been estimated at approximately $€ 21$ million per annum.

The care of people with dementia poses particular challenges for the acute hospital system. The Irish National Audit of Dementia (INAD) ${ }^{16}$, the first national audit of dementia care in Ireland's acute hospitals, which was jointly funded by the Atlantic Philanthropies and the Meath Foundation, found that:-

- $94 \%$ of hospitals have no dementia care pathway in place;

- The majority of wards can provide access at least five days a week to specialist services such as Liaison Psychiatry, Geriatric Medicine, Occupational Therapy, Physiotherapy, Specialist Infection Control and Specialist Palliative Care. There is more limited access to Psychiatry of Old Age, Specialist Continence Services, Psychology and Social Work Services;

- Many hospitals reported no access, or inadequate access, to social workers;

- In many hospitals, psychiatry of old age services are being delivered by communitybased services, who are not formally commissioned to deliver a liaison service to the acute hospital;

- Just over half of hospitals had provided dementia awareness training to doctors (54\%) and nurses (52\%) in the last 12 months. Far fewer hospitals had provided training to healthcare assistants (29\%), allied healthcare professionals (16\%) or support staff (10\%);

- The majority of wards did not have environmental cues to help the person with dementia orientate themselves;

- $86 \%$ of hospitals reported having a named person who takes overall responsibility for complex needs discharge, and this includes people with dementia;

- $50 \%$ of patients admitted from nursing homes were on anti-psychotic medication;

- The reason for use of anti-psychotic medication was only recorded in $50 \%$ of cases;

- Only $32 \%$ of hospitals had developed policies for behaviours that challenge.

People with dementia and other neurological conditions, once admitted to hospital, have poorer outcomes; they spend significantly longer in hospital compared to people aged 65 years and over generally (average length of stay is four times that for other people over 65 years); they are at a high risk of institutionalisation and have an increased risk of mortality.

${ }^{16} \mathrm{http}: / /$ www.ucc.ie/en/media/research/irishnationalauditofdementia/INADFullReportLR.pdf 
It is therefore important that there be clear clinical leadership for dementia in general hospitals. Admission to hospital can cause enormous distress for people with dementia. Delirium is highly prevalent among persons with dementia who are hospitalised and is associated with an increased rate of cognitive deterioration. The active engagement of carers in the care of their loved ones while in acute care is critical.

People with dementia will usually come into contact with a wide range of staff in acute settings. A good awareness of dementia and appropriate skills for dealing with dementia is therefore extremely important for all those who deal with people with dementia in acute hospitals.

During the consultation process, it was highlighted that people with dementia can experience difficulty in having their needs understood and met in hospitals. One explanation offered in this regard was that hospital staff are naturally focused on the acute medical and surgical condition that led to hospitalisation, and that the further or on-going care of the person with dementia is often overlooked.

\section{Long-Term Care Options}

While current health and social policy is focussed on caring for people with dementia in their own homes, there will come a time for many when home care may no longer be feasible or appropriate and when long-term residential care will be the best option. This can particularly arise when 24-hour care is needed or when the person with dementia is living alone.

It is estimated that $34 \%$ of all people with dementia live in residential care, a small proportion of whom (1,000 approx) currently live in specialist care units. The majority of people with dementia currently reside in generic residential care facilities. It is critical that residential standards for Units accommodating people with dementia take appropriate account of the needs of these residents even where the Unit in question is not formally described as specialising in dementia. It is also desirable that people with dementia in residential care facilities should be accommodated as close as possible to their home communities.

Dementia is often accompanied by behavioural and psychological symptoms (BPSD), (sometimes known as behaviours that challenge) such as agitation, aggression, disinhibition, wandering and sleep disturbance and these can occur at any stage of the illness. These behaviours can pose significant challenges for staff and other patients and can be dangerous and distressing for the person with dementia. While these behaviours are common, they can be transient and can sometimes be addressed by non-pharmacological interventions. There are occasions, albeit for a minority of cases, where pharmacological treatments may be required. Pharmacological and non-pharmacological treatments and interventions should go hand-in-hand. People with dementia are more likely to experience psychopathology during the course of their illness and in some cases the use of psychotropic medications is appropriate in conjunction with environmental interventions. However, anti-psychotic drugs should only be used when all other non-pharmacological interventions have first been tried and exhausted (NICE Guidelines). While the use of antipsychotic medications can be effective at controlling BPSD, they are not without risk and if prescribed should be reviewed at regular intervals and discontinued when clinically indicated. 
Specialist Dementia Care Units or other facilities which can accommodate such behaviours, can also play an important part, particularly for those with less severe behavioural challenges.

A recent survey showed that only $6 \%$ of all beds in Specialist Units were for respite and that many of the staff employed in public units had not undergone dementia-specific training. It also showed an uneven distribution of these Specialist Units across the country, with some areas having no such facilities.

\section{Palliative Care for People with Dementia}

Palliative care is defined as an approach that improves the quality of life of patients and their families facing the problems associated with life-threatening illness, through the prevention and relief of suffering. Palliative care may be understood as both a set of principles that underpins an approach to care, and as a type of service that is provided.

Palliative care for people with dementia involves supporting both the person with dementia and their family, and seeking to address and relieve the pain, distress and discomfort associated with end stage dementia. It includes inviting the person and family to participate in making decisions about future care needs, ideally when the person still has capacity to do so. It also provides a framework to anticipate and plan for cognitive loss associated with dementia. More timely diagnosis and disclosure of dementia are, therefore, particularly important in this regard.

Palliative care services are organised into specialist and generalist services that operate in partnership. In Ireland, palliative care services are structured in three levels of ascending specialisation according to the expertise of the staff providing the service:-

- Level one - Palliative Care Approach: Palliative care principles should be appropriately applied by all health care professionals.

- Level two-General Palliative Care: At an intermediate level, a proportion of patients and families will benefit from the expertise of health care professionals who, although not engaged full time in palliative care, have had some additional training and experience in palliative care.

- Level three - Specialist Palliative Care: Specialist palliative care services are those services whose core activity is limited to the provision of palliative care.

The term 'generalist palliative care providers' is used to collectively describe all those services, health and social care providers who possess 'palliative care approach' or 'general' palliative skills (i.e. level one and two services). The role of generalist palliative care providers is fundamental to the provision of high quality care for people with dementia. Indeed, the needs of many patients with dementia can be appropriately and effectively met by generalist palliative care providers alone. However, should an individual experience unstable symptoms or problems of high intensity, complexity and/or frequency as a consequence of their illness, then input from specialist palliative care services is needed to ensure best possible outcomes. 
The National Palliative Care Competence Framework describes the competences required of staff. ${ }^{17}$ All staff caring for people with dementia should possess palliative care approach competences, and selected staff should possess general palliative care competences.

A Feasibility Study carried out jointly by the Irish Hospice Foundation and the Alzheimer Society of Ireland ${ }^{18}$ concludes that palliative care should not only be considered as having a role at end-of-life, but that it extends from point of diagnosis through the trajectory of the illness, to end-of-life and bereavement support for family members. It was considered that this approach would provide people with dementia with opportunities to plan their future care needs and address any fears and apprehensions they may have as their disease progresses.

The inclusion of palliative care principles in services for people with more advanced dementia will help staff in these services to communicate, support and manage a spectrum of care issues ranging from advance care planning to decision making regarding clinical interventions and to specific ethical dilemmas that present for people with dementia and their families.

\section{PRIORITY ACTIONS 7 TO 10 IN SECTION 3 RELATE TO THIS ACTION AREA}

\section{ADDITIONAL ACTIONS}

Subject to overall Government priorities, the Department of Health will as soon as possible formulate proposals and timelines for the regulation of home and community care services for older people.

The Health Service Executive will ensure that the carer assessment component of the Single Assessment Tool (SAT) is used to enable the provision of more targeted supports to carers at an individual level and to enable better planning of services and supports for carers at a national level.

The Health Service Executive will develop and implement a dementia and delirium care pathway, which could be fitted to existing acute, rehabilitative, care of older people, stroke, mental health, palliative care and end-of-life care pathways, to be developed and implemented on a local level in each acute hospital.

The Health Service Executive will assign responsibility in its own facilities, and elsewhere will encourage the assignment of responsibility to, a senior clinician within each hospital to lead the development, implementation and monitoring of the pathway.

Hospitals will be required to ensure that people with dementia have a specific pathway through Emergency Departments and Acute Medical Units that is appropriate to their particular sensory and psychosocial needs.

\footnotetext{
${ }^{17} \mathrm{http} / /$ www.hse.ie/eng/about/Who/clinical/natclinprog/palliativecareprogramme/Resources/ competencyframework.pdf

${ }^{18}$ Building Consensus for the Future - Feasibility Study on Palliative Care for People with Dementia (2012). This report sets out how the palliative needs of people with dementia may be addressed in the health and social care system. http://www.alzheimer.ie/Alzheimer/media/SiteMedia/ImageSlider/Fixed/DPC-Feasibility-Study-reportFINAL.pdf?ext=.pdf
} 
The Health Service Executive will develop guidelines on dementia-friendly ward specification to be taken into account at the design stage of all refurbishments and new builds. Elements to be considered should include safe walking spaces and the use of colour, lighting, signage, orientation cues and space used to promote social interaction.

Hospitals will prioritise the assessment of social and environmental supports to meet the needs of people with dementia and their carers, including appropriate access to social work support.

The Health Service Executive will work to maximise the implementation of the national policy on restraint: Towards a Restraint Free Environment in Nursing Homes. ${ }^{19}$

In the exceptional circumstances where a person with dementia needs acute admission to a psychiatric unit, every effort will be made to secure placement in a suitable old age psychiatry unit.

The Health Service Executive will examine a range of appropriate long-term care options to accommodate the diverse needs of people with dementia, including those with behaviours that challenge. In planning future long-term residential care, the Health Service Executive will take appropriate account of the potential of new residential models, including housing with care, for people with dementia.

\footnotetext{
${ }^{19}$ http://health.gov.ie/blog/publications/towards-a-restraint-free-environment-in-nursing-homes/
} 


\section{SECTION 7 \\ TRAINING AND EDUCATION}

\section{OBJECTIVES}

All staff caring for or interacting with people in adult services across all settings (primary and community; acute; long term and palliative care) should receive on-going training to ensure that they have the necessary skills (including communication skills) and competencies to provide high quality, person-centred care and support.

The development of educational material for health and social care professionals informed by the experiences of people with dementia and their carers.

Training specific to individual professional groups supported by relevant professional bodies.

Informal carers confident and competent to care for their family member at home.

Evaluation of training and educational programmes to ensure that training leads to a change in practice, attitudes and quality of life.

Caring for someone with dementia is challenging, both physically and emotionally. Staff in all relevant care settings need the appropriate skills and competencies to effectively cater for people with dementia of all ages. However, available studies suggest that many staff employed to provide care to people with dementia often have little formal dementia-specific education.

In Primary Care, General Practitioners are often the first healthcare professionals to be consulted when dementia is suspected by patients or their families. Accordingly, GPs should be facilitated and supported to develop the specific knowledge and skills needed to effectively cater for patients presenting with dementia or possible dementia.

In terms of curriculum content, there is broad agreement that the areas for attention need to be:-

- Improved awareness of the benefits that pro-active care can have for people with dementia and their carer;

- Diagnosis and disclosure of dementia;

- Dementia-specific communication skills;

- Ethical and legal knowledge and skills;

- Treatment and ongoing care including pharmacological and non-pharmacological interventions.

The Dementia Services Information and Development Centre (DSIDC)

The DSIDC was set up in 1998 in response to a growing demand for resources for practitioners 
working in the area of dementia and care of older people. The Centre is committed to promoting best practice in all aspects of dementia care and to improving and expanding services for all those affected by dementia.

It provides educational courses and training days to staff providing services to people with dementia in many different care settings around the country. In addition, its research activities contribute to the development of timely, responsive and accessible interventions for people with dementia. It endeavours to influence policy development and to contribute to the design of best practice models for those affected by dementia.

\section{Dementia Education Programmes available through the Health Service Executive and the Alzheimer Society of Ireland}

- Dementia Awareness Educational Programme

- E-Learning Programme on Early identification of memory problems in the older person available on www.hseland.ie

- Dementia Champions Programme (Level 8); DCU \& Health Service Executive

- An information booklet and DVD for home help workers ${ }^{20}$ and health care staff ${ }^{21}$

- Information posters entitled Dementia Delirium Awareness Posters

- 'Responsive Behaviours: Understanding and Supporting the Person with dementia'. Programme. The three DVDs used as part of the programme are available on the Health Service Executive website. ${ }^{22}$

- Dementia Skills Elevator - offering a wide range of education to people with dementia, their carers, public facing services and health and social care professionals. ${ }^{23}$

The educational programmes not only deliver up-to-date information on dementia and dementia care, but are designed to foster an environment where participants can examine and challenge their own unconscious behaviours and beliefs in the area of caring for a person with dementia. To ensure that the education programmes continue to meet the education and information needs of care staff, each programme has an evaluation component built in. In addition, an annual programme evaluation is carried out to identify areas for change/review. These results are presented to the programme review board.

\section{PRIORITY ACTION 1 IN SECTION 3 RELATES TO THIS ACTION AREA}

\section{ADDITIONAL ACTION}

The Health Service Executive will develop appropriate training courses for family and other informal carers in keeping with the priorities highlighted in the National Educational Needs Analysis completed by the Health Service Executive in 2009 and Dementia Skills Elevator $2014 .^{24}$

\footnotetext{
${ }^{20} \mathrm{http} / /$ www.hse.ie/eng/services/Publications/olderpeople/homehelp.pdf

${ }^{21}$ http://www.hse.ie/eng/services/Publications/corporate/NursingMidwifery\%20Services/dementia.html

${ }^{22}$ http://www.hse.ie/eng/about/Who/ONMSD/eductraining/dementiaeducation/ResourcesNursesHealthcareStaff.html

${ }^{23}$ http://dementiaelevator.ie/

${ }^{24} \mathrm{http}: / /$ dementiaelevator.ie/wp-content/uploads/2013/12/Elevator-National-Educational-Needs-Analysis-ReportPrint-Version.pdf
} 


\section{SECTION 8 \\ RESEARCH AND INFORMATION SYSTEMS}

\section{OBJECTIVES}

Funding for clinical, non-clinical (including psychosocial research) and Health Service Executive research on dementia to be a priority.

Better evidence in relation to dementia in Ireland, including:-

- the needs and preferences of people with dementia and their carers and their experiences and care outcomes;

- improved national, regional and local estimates of current and future prevalence across all care settings;

- diagnostic rates, sub-types, use of anti-psychotics, clinical outcomes;

- the need for and use of health and personal social services by people with dementia and their carers;

- health and social care system's responses to the needs of people with dementia and their carers;

- impact of training on quality of care and quality of life;

- evaluation of psychosocial interventions.

Better collection of data and use of evidence to inform health and social system responses to the needs of people with dementia and their carers.

Research on how social factors, financing systems, organisational structures and processes, health technologies and personal behaviours affect access to health care, the quality and cost of health care, and ultimately people's health and wellbeing, can inform the organisation and delivery of future services to achieve optimum health outcomes.

Information on dementia is not currently being recorded on some national databases. This has implications for the provision of evidence-based services and supports and for ensuring that resources are targeted effectively.

Currently, there is no epidemiological study in Ireland which can be relied on for providing accurate numbers on the prevalence of dementia in the Irish population, the characteristics and needs of those with dementia or their carers, and the full range of services and treatments that people with dementia are receiving. The Irish Longitudinal Study on Ageing (TILDA) does not provide clinical or neuropathological confirmation of dementia diagnosis. However, it has generated in-depth epidemiological data on prevalence and incidence of cognitive impairment and dementia as defined by cut-off scores on MMSE and MoCA on 8,000 people in Ireland over age 50, which can inform practice and policy for dementia. 
Research into the causes of and cures for dementia may, in the long-term, make preventative measures and effective treatments a reality. In the short-term and in the absence of cure, people will continue to live with the symptoms of dementia. Non-clinical psychosocial research can provide an improved understanding of how people can live well with dementia.

The public consultation process highlighted the need for research and evidence as a priority for service planning and delivery as well as the need for further investment into research on the cause, cure and care of dementia.

\section{ADDITIONAL ACTIONS}

The Health Service Executive will ensure that data from the Single Assessment Tool (SAT) is factored into research to inform dementia care in Ireland.

The Health Service Executive will take measures to ensure appropriate recording and coding of dementia in primary care and the development of practice-based dementia registers.

Hospitals will take measures to encourage better recording and coding of a primary or secondary diagnosis of dementia in hospital records and charts to ensure that admissions, re-admissions, lengths of stay and discharge for people with dementia are captured on HIPE (Hospital In-Patient Enquiry). 


\section{SECTION 9 \\ LEADERSHIP}

The National Dementia Strategy seeks to improve the quality of life for people with dementia and their carers and this will be done by setting a direction for change over an extended period, based on a set of desired outcomes and associated actions.

The Strategy aims to lead to systemic changes - i.e. changing societal attitudes to dementia and influencing frontline practice within large systems including Primary and Community Care, Mental Health, Hospital and Long-Term Care.

At a policy level, the Department of Health will report on progress to the Cabinet Committee on Social Policy, which is chaired by An Taoiseach. A National Dementia Strategy Monitoring Group will be established to monitor progress towards implementation of the Strategy.

The implementation of this Strategy will take place alongside the delivery of other significant health and social care policies, notably the Health Service Executive reform programme, the National Carers' Strategy ${ }^{25}$, the National Positive Ageing Strategy ${ }^{26}$ and Healthy Ireland ${ }^{27}$.

\section{PRIORITY ACTIONS 12 TO 14 IN SECTION 3 RELATE TO THIS ACTION AREA}

\section{ADDITIONAL ACTIONS}

Clear and effective management structures will be established within the Health Service Executive to provide leadership at a system level for the implementation of the Strategy.

The Department of Health and the Health Service Executive will develop performance indicators to measure progress in implementing the Strategy. The Department of Health will also conduct a mid-term review of progress in 2016.

\footnotetext{
${ }^{25}$ http://health.gov.ie/blog/publications/national-carers-strategy/

${ }^{26} \mathrm{http}$ //health.gov.ie/blog/publications/national-positive-ageing-strategy/

${ }^{27}$ http://www.hse.ie/eng/services/publications/corporate/hieng.pdf
} 


\section{Appendix 1}

Terms of Reference for the Advisory Group established to assist in the development of the National Dementia Strategy

\section{Background}

The Government has given a commitment to develop a National Dementia Strategy by the end of 2013 to increase awareness, ensure early diagnosis and intervention, and development of enhanced community based services.

The development of the Strategy is taking place during a period of very challenging financial circumstances and the Strategy will therefore have to have due regard to the constraints imposed by the budgetary situation while also encouraging changes in work practices, processes and realignment of resources which will deliver real results both for those who have dementia and for their families, carers, community and society and for the professionals managing and providing the services and supports for people with dementia.

As indicated in the Programme for Government, the purpose of the Strategy will be:-

- to increase awareness;

- ensure early diagnosis and intervention;

- ensure development of enhanced community based services.

\section{Establishment of an Advisory Group}

The Minister for Health has decided to establish an Advisory Group to assist in the drafting of a National Dementia Strategy for his consideration.

\section{Meetings of the Advisory Group}

Meetings will normally take place in the Department of Health office in Hawkins House. The Advisory Group may invite persons or organisations who are not members of the Advisory Group to present to the Group either in person or in writing or to participate on subgroups along with Advisory Group members to consider specific issues and to make recommendations for consideration by the full Advisory Group.

\section{Role of the Advisory Group}

In preparing a draft Strategy for the Minister's consideration, the Advisory Group will take account of the following:-

1. the principles, goals and objectives established in the National Positive Ageing Strategy;

2. the fact that Ireland will experience an unprecedented ageing of the population in the first half of the twenty-first century, with the greatest increases expected in the over- 80 age group; 
3. that for those with dementia as well as for others, well-being is a multi-dimensional concept that is determined not only by health services but by a range of interconnected social, economic and environmental factors. A "whole of Government" approach should therefore be applied;

4. that the draft Strategy should include measures to:-

- promote greater public awareness of the nature of dementia, of its symptoms, causes and risk factors, latest information on how risk can be reduced, and of what treatments, services and supports are available to those diagnosed with dementia and their families and carers;

- facilitate effective preparation and planning for their futures by those diagnosed with dementia;

- recognise and facilitate the wish of many people with dementia to stay in their own homes and communities for as long as possible using early intervention, integrated care pathways, case management and enhanced community services;

- simplify and streamline to the greatest extent possible the administrative processes applied to those seeking State services/supports for or on behalf of persons with dementia;

- recognise and support the role of carers in accordance with the provisions of the National Carers Strategy, including in the areas of advice, training, and respite services.

5. the current economic situation in Ireland and the implications of that for the availability of financial and other resources. 


\section{Appendix 2}

Estimates of number of people with dementia in Ireland, 2011

\begin{tabular}{|c|r|r|r|r|r|}
\hline \multirow{2}{*}{ Age groups } & \multicolumn{2}{|c|}{$\begin{array}{c}\text { Total Population } \\
\text { (Census 2011) }\end{array}$} & \multicolumn{2}{c|}{$\begin{array}{c}\text { Persons with dementia (estimated using } \\
\text { EuroCoDe (2009) } \begin{array}{c}\text { age/gender-related } \\
\text { dementia prevalence rates) }\end{array}\end{array}$} \\
\hline & Men & \multicolumn{1}{c|}{ Women } & Men & \multicolumn{1}{c|}{ Women } & Total \\
\hline $30-59$ years & 953,715 & 959,396 & $1907^{1}$ & 9591 & 2,866 \\
\hline $60-64$ years & 109,869 & 108,917 & 220 & 980 & 1,200 \\
\hline $65-69$ years & 86,298 & 87,340 & 1,553 & 1,223 & 2,776 \\
\hline $70-74$ years & 63,476 & 67,714 & 2,031 & 2,573 & 4,604 \\
\hline $75-79$ years & 46,631 & 55,405 & 3,264 & 4,211 & 7,475 \\
\hline $80-84$ years & 28,423 & 41,690 & 4,121 & 6,837 & 10,958 \\
\hline $85-89$ years & 13,591 & 26,296 & 2,841 & 7,494 & 10,335 \\
\hline $90-94$ years & 4,155 & 10,722 & 1,213 & 4,761 & 5,974 \\
\hline $95+$ & 740 & 2,912 & 240 & 1,421 & 1,661 \\
\hline Total & $1,306,898$ & $1,360,392$ & 17,390 & 30,459 & 47,849 \\
\hline
\end{tabular}

Source: CSO (2011) Census of Population - Profile 2: Older and Younger: An Age profile of Ireland Tables 7A-7C, pp. 47-49; Persons with dementia estimated by application of EuroCoDe gender/agespecific prevalence rates.

Notes: As EuroCoDe does not specify early onset prevalence rates, EURODEM early onset dementia prevalence rates were used instead. (Source: Pierce, M. et al. (forthcoming). Prevalence and Projections of Dementia in Ireland, 2011. Genio Ltd., Mullingar). 

Department of Health

Hawkins House

Hawkins Street

Dublin 2

Ireland

Tel: +353 (0)1 6354000

www.health.gov.ie 